
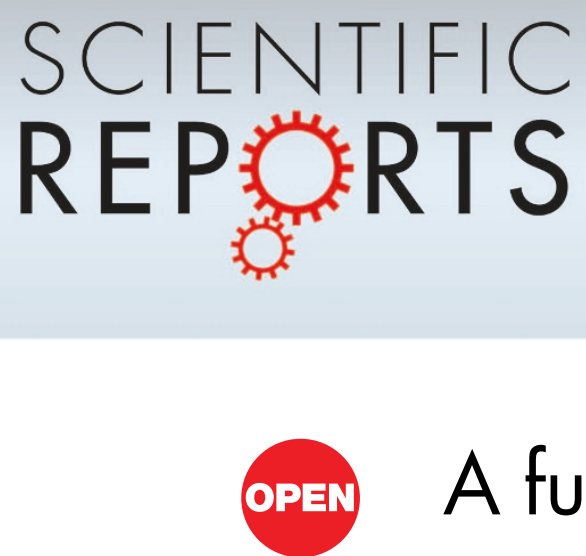

\title{
A functioning artificial secretory cell
}

\author{
Lisa Simonsson ${ }^{*}$, Michael E. Kurczy ${ }^{2 *}$, Raphaël Trouillon ${ }^{3}$, Fredrik Hook ${ }^{1} \&$ Ann-Sofie Cans ${ }^{2 *}$
}

SUBJECT AREAS:

SYNTHETIC BIOLOGY

ANALYTICAL CHEMISTRY

BIOLOGICAL MODELS

SYNAPTIC TRANSMISSION

Received

18 July 2012

Accepted

25 October 2012

Published

8 November 2012

Correspondence and requests for materials should be addressed to

A.-S.C. (cans@

chalmers.se)

* These authors contributed equally to this work.
'Department of Applied Physics, Chalmers University of Technology, Gothenburg, Sweden, ${ }^{2}$ Department of Chemical and Biological Engineering, Chalmers University of Technology, Gothenburg, Sweden, ${ }^{3}$ Department of Chemistry, Gothenburg University, Gothenburg, Sweden.

We present an amperometric study of content release from individual vesicles in an artificial secretory cell designed with the minimal components required to carry out exocytosis. Here, the membranes of the cell and vesicles are substituted for protein-free giant and large unilamellar vesicles respectively. In replacement of the SNARE-complex, the cell model was equipped with an analog composed of complimentary DNA constructs. The DNA constructs hybridize in a zipper-like fashion to bring about docking of the artificial secretory vesicles and following the addition of $\mathrm{Ca}^{2+}$ artificial exocytosis was completed. Exocytotic events recorded from the artificial cell closely approximate exocytosis in live cells. The results together with simulations of vesicular release demonstrate that the molecular flux in this model is attenuated and we suggest that this is the result of restricted diffusion through a semi-stable fusion pore or a partitioning of the signalling molecule out of the fused vesicle membrane.

xocytosis is a complex process involving a wide variety of lipids, proteins (the most important being the soluble N-ethylmaleimide-sensitive factor attachment protein receptor (SNARE) proteins), the molecules to be secreted and $\mathrm{Ca}^{2+}$ ions. Here we present a bottom-up approach to create an artificial secretory cell designed to model exocytosis. The synthetic character of this model allows for the tuning of molecular complexity in a manner that is not possible in a living cell.

The SNARE complex ${ }^{2}$, which is responsible for stimulated exocytosis in living cells, was replaced by an analogue composed of short complimentary DNA strands. These DNA constructs have previously been shown to hybridize in a zipper-like fashion and induce vesicle-vesicle fusion in suspension ${ }^{1,3,4}$ as well as fusion of vesicles to planar supported ${ }^{5}$ and tethered lipid bilayers ${ }^{6}$. These studies have shown that the DNA zipper, in contrast to DNA constructs that only tether lipid membranes ${ }^{5}$, is an effectual mimic of the coiled coil formation of SNARE proteins. However, it was not until the recent work on tethered membranes that content release on the level of single vesicles could be detected for the first time ${ }^{6}$, although the limited sensitivity and time resolution of fluorescence imaging could only aid in stating that pore opening occurs within less than $10 \mathrm{~ms}$.

We have in this work employed amperometry with sub-millisecond time resolution in a synapse configuration to measure the artificial exocytotic events from this system. Exocytotic events in living secretory cells have been routinely detected and quantified using amperometric recordings ${ }^{7,8}$. Additionally the characteristic amperometric spike corresponding to an individual release event can be used to reveal mechanistic details about the final stages of the exocytosis ${ }^{8}$. The combined sub-millisecond time resolution and single vesicle sensitivity of amperometry used in this work has provided a unique ability to directly compare the rudimentary action of vesicle content release from an artificial secretory cell to the more complex exocytotic release at live cells.

\section{Results}

The minimal system presented in this work is composed of two basic structures, the artificial cell's plasma membrane and the artificial secretory vesicles. The artificial plasma membrane was provided by a giant unilamellar vesicle (GUV) with a diameter of $\sim 25-50 \mu \mathrm{m}$ formed from soybean lipid extract. The target-SNAREs ( $\mathrm{t}$ SNAREs) syntaxin and SNAP-25 are substituted for target-cholesterol-DNA strands ( $\mathrm{t}-\mathrm{CH}-\mathrm{DNA}$ labelled b/b', in Figure 1a), which were electroinserted ${ }^{9}$ into the interior of a GUV using a microinjection pipette ${ }^{9,10}$ (Figure 1a and b). Hydrophobicity drives the cholesterol moieties into the hydrophobic core of the membrane, which results in the anchoring of the $\mathrm{t}-\mathrm{CH}-\mathrm{DNA}^{11}$. The result is that the inner leaflet of the artificial secretory cell's plasma membrane resembles the inner leaflet of the plasma membrane of a living cell which is decorated with t-SNAREs.

Artificial secretory vesicles were made from large unilamellar vesicles (LUVs, mean diameter $\sim 200 \mathrm{~nm}$ ) and filled with catechol as a neurotransmitter analogue. Cholesterol-DNA strands complementary to the t-CH-DNA (labeled a/a', in Figure 1a), were incorporated into the outer leaflet of the vesicle compartment and served as vesicle-SNARE (v-CH-DNA) synaptobrevin analogues. The artificial secretory vesicles were electroinjected into 


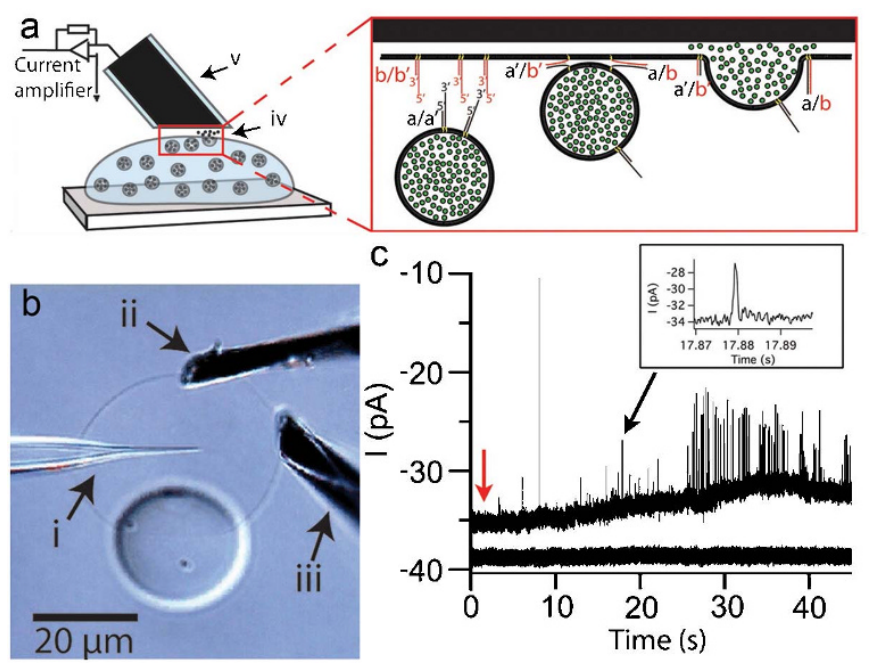

Figure $1 \mid$ (a) Schematics of a GUV containing LUVs filled with catechol. Catechol release (iv) upon LUV fusion to the GUV membrane is detected by a carbon fiber electrode (v). The expanded view provides details of DNA mediated exocytosis. Injection and self-insertion of CH-DNA b/b' (red) into the GUV membrane was followed by a second injection of catecholfilled LUVs decorated with the complementary CH-DNA a/a' (black). The DNA strands hybridize in a zipper-like fashion and bring about fusion of the two membranes. Cartoons are not drawn to scale. (b) Nomarski image of a GUV, with a multilamellar bleb, attached as a lipid reservoir, a microinjection pipette electroinserted into the interior of a GUV (i), electrode for electroinsertion (ii) and a $5-\mu \mathrm{m}$ diameter amperometric electrode, beveled to a $45^{\circ}$ angle (iii). (c) Amperometric recording of repeated exocytosis events at the secretory cell model, the apparent delay in release in due to reposition of the electrode (upper trace). The lower trace represents amperometric monitoring after injection of vesicles that do not display $\mathrm{CH}-\mathrm{DNA}$. The arrow indicates the start of $\mathrm{Ca}^{2+}$ addition. The inset shows a single exocytosis event spike at an expanded time scale.

the GUV where they presumably dock to the DNA-labelled GUV membrane. Vesicle fusion was then initiated by $\mathrm{Ca}^{2+}$ entry into the GUV by a further injection, hence simulating the critical aspects of exocytosis in live cells. See Methods for details on vesicle production and lipid composition and Supplementary Information for details on the CH-DNA design.

The LUV membranes were prepared with a lipid composition that previously was found to be very fusogenic ${ }^{3}$. In this study, we found that like in living cells, $\mathrm{Ca}^{2+}$ ions are also important for initiation of this process. The calcium dependent exocytosis in the artificial secretory cell is a consequence of filling the vesicles with catechol, as very little spontaneous fusion in absence of $\mathrm{Ca}^{2+}$ was observed from catechol filled vesicles (see Supplementary Information). It appears that the act of filling the vesicles with catechol affects them in a way that hinders exocytosis while the addition of $\mathrm{Ca}^{2+}$ lowers the energy barrier for fusion. This observation has allowed us to design a calcium-dependent artificial secretory cell.

A $5 \mu \mathrm{m}$ carbon fibre microelectrode placed against the outer leaflet of the GUV membrane (Figure 1a and b) completed the artificial synapse with an estimated cleft of $\approx 300 \mathrm{~nm}^{12}$. Upon the addition of $\mathrm{Ca}^{2+}$ to the interior of the GUV, a train of amperometric spikes was observed (Figure 1c), where each amperometric spike corresponded to an individual vesicle release event. The apparent delay of the release events in figure $1 \mathrm{c}$ is attributed to a repositioning of the electrode following the addition of calcium. A negative control using LUVs lacking the $\mathrm{v}$-CH-DNA did not show any amperometric spikes upon $\mathrm{Ca}^{2+}$ addition (lower trace in Figure 1c). Accordingly, we conclude that the combination of a SNARE analogue and the presence of calcium are required for this functioning artificial secretory system.
Statistics of current spike parameters, such as rise time $\left(t_{\text {rise }}\right)$, calculated as the time it takes for the current to rise from $25 \%$ of its maximum value to $75 \%$, fall time $\left(t_{\text {fall }}\right)$, calculated as the time it takes for the current to fall from $75 \%$ to $25 \%$ of the maximum value, and the full width at half maximum amplitude (FWHM) can be used to investigate individual fusion events. The FWHM and $t_{\text {fall }}$ are characteristic of transmitter flux ${ }^{8}$ and fusion pore diameter (see below). Characteristics of the fusion pore expansion are traditionally inferred from $t_{\text {rise }}{ }^{13}$. However, it cannot be excluded that the fusion pore continues its expansion after the maximum current is reached. The high temporal resolution afforded by amperometric recordings have made it possible to infer details about fusion pore opening and content release of the artificial cell.

A comparison of the normalized average spikes measured at the secretory cell model and at a Rat pheochromocytoma (PC12) cell is shown in Figure 2a. This comparison reveals that the peaks are quite similar in terms of shape characteristics. The temporal statistics for the artificial cell model and comparative data from measurements at a PC12 cell are shown in Figure $2 b$ and $c$ and the averages of these values are summarized in Table 1. As seen in Figure 2a and in the histograms (Figure $2 b-c$ ), fusion events recorded from the secretory cell model are slightly faster in all respects when compared to the events measured at living PC12 cells. Diffusion during exocytosis in living cells is apparently restricted to a greater degree than that observed in our lipid-based cell model.

\section{Discussion}

Electrochemically measured release from large dense core vesicles (LDCV), the most common secretory vesicle found in PC12 cells, is known to be slower than would be expected from a purely diffusionlimited system ${ }^{8}$. Partitioning of neurotransmitters into the dense core has been shown to be an important mechanism for maintaining high concentrations within secretory vesicles ${ }^{14}$. Indeed, this strong interaction has been proposed to cause the observed hampering of content release ${ }^{8}$. In our artificial secretory cell we expected diffusion limited release kinetics, which by considering Fick's first law, corresponds to sub-millisecond events. The events measured from the artificial secretory cell occur over several milliseconds (Figures 1c and $2 \mathrm{a}$ ), suggesting that the diffusion of catechol from the vesicle is indeed restricted - despite the absence of a dense core.

One possibility for the observed reduced flux is that the secretory molecules pass through a limited area defined by the diameter of a fusion pore connecting the secretory vesicle to the plasma membrane. To investigate this possibility a rudimentary simulation of the fusion pore was employed (see Supplemental Information for model details). The average measured peak from the cell model is plotted together with simulated peaks in Figure 3. The peak amplitude predicted for the unrestricted diffusion of catechol from a $165 \mathrm{~nm}$ (the largest fraction of LUVs, see Supplementary Figure 3) bolus (approximately 40,000 molecules) across the artificial synapse (Figure 3 ) is an order of magnitude larger than the average measured peak. Furthermore, the predicted FWHM was observed to be an order of magnitude smaller than the measured value. Therefore, to approximate the measured peak, the flux of catechol was modelled by assuming that an aperture ( 3 or $6 \mathrm{~nm}$ ) exists between the vesicle and the synapse (Figure 3 ). This additional feature simulates a fusion pore, which may or may not limit the flux of neurotransmitters during exocytosis in cells ${ }^{15}$. This pore simulation gives a hint to what may control the release of LUV content in our model system. From the results presented in Figure 3, it is clear that a $3 \mathrm{~nm}$ fusion pore would give rise to a spike similar to the measured average peak from the artificial secretory cell. Data presented in the Supplementary Information however suggest that $t_{\text {fall }}$, is better represented by a $5 \mathrm{~nm}$ pore, suggesting that the pore expands during the later stage of LUV content release. Integrating an expanding aperture into this calculation could potentially solve the fall time discrepancy. 
a

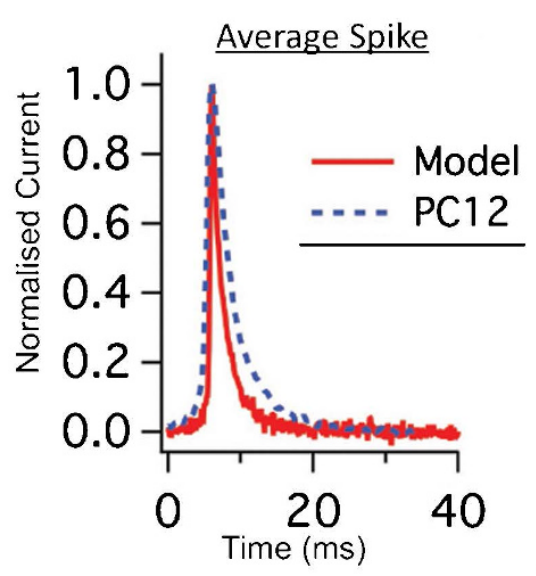

b Cell Model

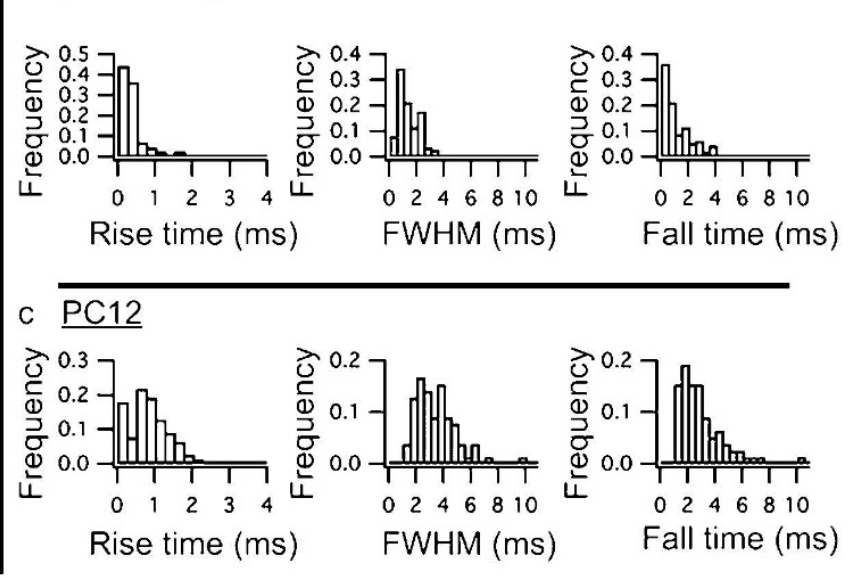

Figure $2 \mid$ (a) Normalized average exocytosis event spikes for the artificial cell model (red) and for a PC12 cell (blue). (b) and (c) Histograms showing distributions of spike kinetics for the artificial cell model $\left(\mathrm{b}, n_{\mathrm{model}}=113\right)$ and a PC12 cell $\left(\mathrm{c}, n_{\mathrm{PC12}}=78\right)$. The displayed spike features are the rise time, calculated as the time it takes for the current to rise from $25 \%$ of its maximum value to $75 \%$, the full width at half maximum (FWHM) current value and the fall time, calculated as the time it takes for the current to fall from $75 \%$ of its maximum value to $25 \%$. The average values of these parameters are listed in Table 1.

However, for the scope of the present work, which focus on the mere fact that exocytosis seems hindered also in a fully artificial system, the static pore model provides sufficient information.

In addition to extrusion thorough a fusion pore, an interaction between catechol and the membrane might have altered the physical properties of the artificial secretory vesicles and might restrict the diffusion of catechol from the fused LUVs. Based on hydrophobicity, catechol is not thought to significantly accumulate in lipid membranes $^{16}$. However recent work suggests ${ }^{17}$ that the catecholamines dopamine and L-Dopa do accumulate in mixed composition lipid bilayers based on hydrogen bonding of amine and hydroxyl groups. Experimentally, it was shown that the interaction is manifested as an increase in lateral pressure in the membrane ${ }^{17}$. It is therefore possible that hydrogen bonding of the hydroxyl groups on the catechol molecule might influence the membrane such that it deviates from the initial fusogenic state. In fact, using a nanoparticle tracking analyser we observe that catechol filled vesicles are larger (mean diameter $\approx$ $200 \mathrm{~nm}$ ) than vesicles typically obtained from extrusion though a $100 \mathrm{~nm}$ pore sized polycarbonate filter ${ }^{18}$. A strong interaction between catechol and the LUV membrane, similar to the proposed dense core interaction mentioned above, was investigated with quartz crystal microbalance with dissipation (QCM-D) ${ }^{19}$ measurements (See Supplementary Information). No irreversible interaction was observed but the time scale of the experiment made it difficult to rule out interactions with sub-second kinetics. Thus it is possible that the interaction proposed above $\mathrm{e}^{17}$ could contribute to the slower than diffusion flux of catechol.

The detailed mechanistic role of $\mathrm{Ca}^{2+}$ in the model system is considered beyond the scope of this report, but it certainly warrants discussion. It is generally believed that $\mathrm{Ca}^{2+}$ bridges lipids of negative charge ( $\sim 25 \%$ in soybean lipid extract) as well as zwitterionic lipids such as DOPC ${ }^{20,21}$. This interaction possibly enables lipid molecules to overcome repulsive electrostatic and hydration forces, as a first

\begin{tabular}{|c|c|c|c|}
\hline & FWHM [ms] & Rise $(25-75)[\mathrm{ms}]$ & Fall $(75-25)$ [ms $]$ \\
\hline $\begin{array}{l}\text { PC1 } 2 \text { cell } \\
\text { Model }\end{array}$ & $\begin{array}{l}3.4 \pm 1.5 \\
1.4 \pm 1.0\end{array}$ & $\begin{array}{l}0.86 \pm 0.68 \\
0.42 \pm 0.80\end{array}$ & $\begin{array}{l}2.9 \pm 1.6 \\
1.4+1.5\end{array}$ \\
\hline
\end{tabular}

step in the membrane fusion process ${ }^{20}$. Calcium ions might also screen the negatively charged backbones of the $\mathrm{CH}-\mathrm{DNA}$, which might result in the assembly of a larger number of $\mathrm{CH}$-DNA strands within the region of membrane contact ${ }^{2}$. With these speculations about the role of calcium, it also makes sense to consider the possible influence of catechol and how it might affect the fusibility of the LUVs. In addition to the proposed membrane-catechol interaction mentioned above, we have imposed an osmotic gradient by attempting to fill the vesicles with a large concentration of catechol (200 mM). Osmotic pressure is expected to lead to swelling and possibly leakage and rupture of the vesicles during preparation. Interestingly, this effect may also be responsible for the larger than expected measured LUV diameters pointed out above. The low loading efficiency $(\approx 15 \%)$ calculated based on the measured charge also supports the possibility that the osmotic pressure has affected the artificial secretory vesicles. This apparent change in the properties of the vesicles may have also rendered them less fusogenic as compared to empty vesicles ${ }^{4}$. The observation that catechol causes $\mathrm{Ca}^{2+}$-triggered fusion of the membranes, is a serendipitous technical issue that has made it possible to trigger fusion with an injection pipette by mimicking $\mathrm{Ca}^{2+}$ translocation through membrane channels.

These results and simulations suggest that, the kinetics of release is affected by the lipid membrane, either by diffusion through a nanometer sized pore or by partitioning of catechol out of the membrane. These two mechanisms involving the membrane may also apply to measurements from LDCV. In cells we find that chemical release is comparatively slower than the artificial secretory cell. In living cells, this greater reduction of flux during exocytosis may originate from reduced diffusion coefficients in the matrices associated with the cell. In light of the data presented here, a larger resistance to pore expansion in the living cell, or the previously cited ${ }^{17}$ strong interaction between dopamine and the lipid membrane might be two additional possible mechanisms.

Finally, we would like to stress that in amperometric recordings of secretory release from living cells, pre-spike features initiated immediately before main single oxidation current spikes, also called prespike amperometric "feet" 22 , are frequently observed. Combined patch-clamp and amperometry have confirmed secretion through an initial nanometric fusion pore ${ }^{22,23}$, prior to further expansion of the pore and complete fusion, as the origin of these pre-spike feet. In a typical amperometric recording of stimulated PC12 cells, approxi- 


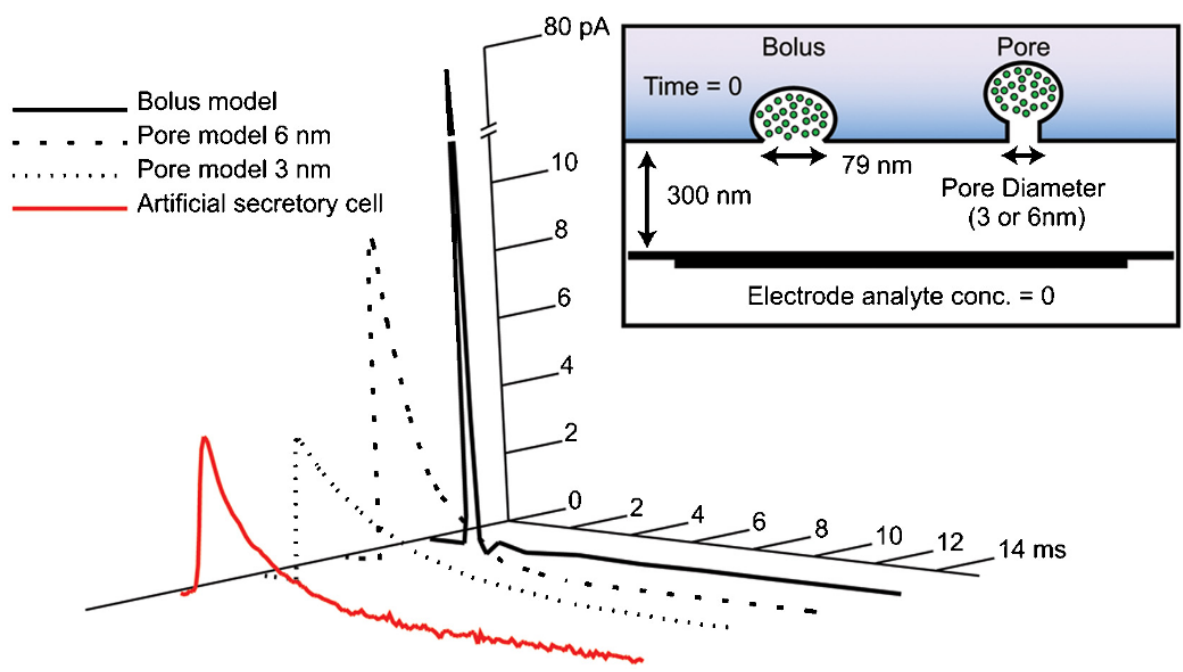

Figure 3 Spikes predicted for the diffusion of catechol across the model synapse originating from either a bolus or from a pore (black dotted lines) and an average of the measured spikes taken from a single GUV (red) the inset depicts time $=0$ for both the pore model and the bolus model.

mately $30 \%$ of the events show pre-spike feet. The spikes from our secretory cell model were scanned for possible so-called pre-spike feet using a routine obtained from David Sulzer's group ${ }^{24}$ and criterion for feet, as stated by Amatore et al..$^{22}$. In the artificial cell, less than $1 \%$ of the recorded spikes showed possible feet; and, most of these were so small that they were difficult to resolve from the noise. Whereas lipids alone are capable of generally explaining secretion kinetics, some other component not accounted for in this model such as the SNARE complex and/or the vesicle dense-core matrix seem to be needed for slow secretion through an initial stable fusion pore.

In conclusion, using an artificial secretory cell we have shown that lipids alone, possibly in combination with their interaction with catechol, appear capable of controlling the kinetics of vesicle secretion, which may give some insight to observations made for living cells. The finding that lipids seem critical for the process relates to previous studies of live cells, which upon incubation with different lipid species showed altered kinetic during exocytosis ${ }^{25,26}$. We believe that this bottom-up cell model, where it is possible to increase complexity in a step-wise manner, will constitute a powerful tool for studies of exocytosis on the molecular level. Combining this model with amperometry, a well-established method for studies of exocytosis, makes it possible to analyse events with sub-millisecond temporal resolution, which is difficult to achieve with fluorescence methods. Finally, an artificial secretory cell has been constructed from the minimal components needed to mimic exocytosis. This could be an important step toward developing a minimal synthetic living system.

\section{Methods}

Materials. Soybean lecithin (polar lipid extract), 1,2-dioleoyl-sn-glycero-3phosphocholine (DOPC), 1,2-dioleoyl-sn-glycero-3-phosphoethanolamine (DOPE) and cholesterol were purchased from Avanti Lipids Inc., Alabaster, AL. Oligonucleotides were purchased from Eurogentec S.A., Seraing, Belgium; sequences: 5'-TGG-ACA-TCA-GAA-ATA-AGG-CAC-GAC-GGA-cholesterol-3' (a); 5' cholesterol-TCC-GTC-GTG-CCT-3' (a'); 5'-ACC-TGT-AGT-CTT-TAT-TCCGTG-GTC-CCT-cholesterol-3' (b); 5' -cholesterol-AGG-CAG-CAC-GGA-3' (b'). Tris(hydroxymethyl)-aminomethane hydrochloride (Tris) was from VWR International, Stockholm, Sweden. Pyrocatechol (catechol), calcium chloride $\left(\mathrm{CaCl}_{2}\right)$, sodium chloride $(\mathrm{NaCl})$, ethylenediaminetetraacetic acid (EDTA), methanol and chloroform were from Sigma Aldrich, Steinheim, Germany. Hydrogen chloride $(\mathrm{HCl})$ was from Merck, Darmstadt, Germany. Tris(hydroxymethyl)-aminomethane free base (Trisma base), potassium phosphate tribasic $\left(\mathrm{K}_{3} \mathrm{PO}_{4}\right)$, potassium phosphate dibasic $\left(\mathrm{K}_{2} \mathrm{HPO}_{4}\right)$, potassium phosphate monobasic $\left(\mathrm{KH}_{2} \mathrm{PO}_{4}\right)$ and magnesium sulfate $\left(\mathrm{MgSO}_{4}\right)$ were purchased from Sigma Aldrich, Stockholm Sweden.

Preparation of LUVs. LUVs were composed of DOPC/DOPE/cholesterol (39:21:40 mol\%). Lipids were dissolved in a 1:1 chloroform/methanol mixture. Lipid vesicles were prepared by evaporation of the solvent under $\mathrm{N}_{2}(>1 \mathrm{~h})$, followed by hydration for $>30 \mathrm{~min}$ in buffer containing catechol $(200 \mathrm{mM}$ catechol, $10 \mathrm{mM}$ Tris/HCl pH
8.0, $100 \mathrm{mM} \mathrm{NaCl}, 1 \mathrm{mM}$ EDTA). The lipid solution was then subjected to five freeze/thaw cycles to promote entrapment of the catecholamine into the vesicles ${ }^{27}$. This was accomplished by alternating a Falcon tube containing the lipid solution between a liquid $\mathrm{N}_{2}$ and warm water bath. The mixture was then extruded through $100 \mathrm{~nm}$ polycarbonate membranes (Whatman, Maidstone, UK) 11 times. The total lipid concentrations were $0.5 \mathrm{mg} / \mathrm{ml}$. The LUV suspension was stored at $4^{\circ} \mathrm{C}$. Nonencapsulated catechol was removed from the LUV suspension using illustra ${ }^{\mathrm{TM}}$ Microspin ${ }^{\mathrm{TM}}$ S-200 HR columns (GE Healthcare, Buckinghamshire, UK.). The LUVs were functionalized with v-CH-DNA at an average LUV-to-DNA ratio of $1: 40$ or $1: 20$, in a buffer solution ( $10 \mathrm{mM}$ Tris/ $\mathrm{HCl} \mathrm{pH} \mathrm{7.4,100} \mathrm{mM} \mathrm{NaCl,} 1 \mathrm{mM} \mathrm{EDTA})$ to a total lipid concentration of $50 \mu \mathrm{g} / \mathrm{ml}$ prior to each experiment. Self-incorporation of the DNA strands was allowed to take place for at least 15 min prior to injection into the GUV. LUVs were never used for more than two days. Size distributions of the catechol-filled LUVs were measured after preparation and prior to injection into GUVs, using a nanoparticle tracking analyzer (NanoSight, Amesbury, UK). The average diameter was determined to $\sim 200 \mathrm{~nm}$.

GUV preparations and manipulations. Surface-immobilized GUVs were prepared from soybean polar lipid extract dissolved in chloroform as previously described ${ }^{10,28}$. The lipid extract was dried using a rotation evaporator (Büchi, Switzerland). The lipid film was then rehydrated with rehydration buffer ( $5 \mathrm{mM}$ Trizma base $\mathrm{pH} 7.4,15 \mathrm{mM}$ $\mathrm{K}_{3} \mathrm{PO}_{4}, 30 \mathrm{mM} \mathrm{KH}_{2} \mathrm{PO}_{4}, 15 \mathrm{mM} \mathrm{K}_{2} \mathrm{HPO}_{4}, 0.5 \mathrm{mM}$ EDTA and $1 \mathrm{mM} \mathrm{MgSO}_{4}$ ) and stored at $4{ }^{\circ} \mathrm{C}$ for approximately $24 \mathrm{~h}$ and then sonicated on ice for $15 \mathrm{~min}$. Three $\mu \mathrm{L}$ of the lipid suspension was then micropipetted onto a glass coverslip (Menzel-Gläser, Braunschweig, Germany; $24 \mathrm{~mm} \times 60 \mathrm{~mm}$, no. 1) and dehydrated in a vacuum desiccator. The slides were transferred to the microscope for experiments and the film was again hydrated using buffer solution $(10 \mathrm{mM}$ Tris/HCl pH 7.4, $100 \mathrm{mM} \mathrm{NaCl}$, $1 \mathrm{mM}$ EDTA). This method spontaneously produced mainly unilamellar vesicles attached to a multilamellar liposome that operates as a lipid reservoir during the experiment. Unilamellarity is a requirement for the measurements. Studying the giant vesicles in a DIC microscope helps in choosing giant vesicles that have a thinner membrane and hence larger chance of being unilamellar.

Further manipulation of the GUVs was performed using injection pipettes, a counter electrode and a Femtojet microinjector (Eppendorf/Brinkmann Instruments, Hauppauge, NY). Injection pipettes were pulled from borosilicate glass capillaries (1.0 mm O.D., $0.78 \mathrm{~mm}$ I.D., Sutter Instrument Co., Novato, CA), using a commercial pipette puller (Model PE-21, Narishige Inc., London, UK) and were backfilled with solution before microinjections. A counter electrode for electroporation, constructed from a pre-fabricated 5- $\mu \mathrm{m}$ carbon fiber tip (ProCFE, Dagan Corp, Minneapolis, MN), was placed on the opposite side of the liposome from the injection pipette. The pipette was then electro-inserted into the liposome with the aid of a voltage pulse generated by a constant voltage isolated stimulator (DS2A-Mk. II, Digitimer, Inc., Hertfordshire, UK). CH-DNA and vesicle solutions were ejected into the GUV by means of a Femtojet microinjector.

Bright-field microscopy. Bright-field imaging was monitored with an Olympus IX71 microscope (Olympus, Melville, NY) with a 40X oil objective (Olympus, UApo/ $34040 \mathrm{X}$ oil iris, NA 1.35). An Olympus SC20 digital color camera interfaced to a personal computer with Cell-A software (Olympus, Hamburg, Germany) was used for visual observation of the experiments.

Electrode fabrication and electrochemical data acquisition. Carbon fiber microelectrodes were prepared by aspirating single $5-\mu \mathrm{m}$ diameter carbon fibers into borosilicate glass capillaries (1.2 mm O.D., $0.69 \mathrm{~mm}$ I.D., Sutter Instrument Co., 
Novato, CA). The filled capillaries were then pulled to a taper using a commercial micropipette puller (Model PE-21, Narishige, Inc., London, UK) and epoxy (Epoxy Technology, Billerica, MA) was used to seal the glass-carbon fiber junction of the electrode. The electrode tips were cut using a scalpel and polished at a $45^{\circ}$ angle on a diamond dust-embedded micropipette beveling wheel (Model BV-10, Sutter Instrument Co., Novato, CA). Electrodes were tested in a solution of $100 \mu \mathrm{M}$ dopamine before experiments and only electrodes with stable I-E curves were used. For measurements, the working electrode was placed against the GUV membrane using a piezo micropositioner (PCS-750/1000, Burleigh Instruments, Fishers, NY) and held at $+750 \mathrm{mV}$ versus a silver/silver chloride reference electrode (Scanbur, Sweden) using an Axon 200B potentiostat (Molecular Devices, Sunnyvale, CA). The output was digitized at $10 \mathrm{kHz}$ ( $5 \mathrm{kHz}$ was used for measurements at PC12 cells) and filtered at $2 \mathrm{kHz}$ via an internal four-pole lowpass Bessel filter. Analysis of the amperometric data was done using the Quanta Analysis routine ${ }^{21}$ written in Igor Pro (Wave Metrics, Lake Oswego, Oregon).

Cell culture and cell measurements. PC12 cells were obtained from the American Type Culture Collection (Manassas, VA) and maintained as described previously ${ }^{29}$. Cells were grown on mouse collagen-IV-coated dishes (Becton Dickinson, Bedford, MA) in RPMI-1640 medium supplemented with $10 \%$ equine and $5 \%$ fetal bovine serum in a $7 \% \mathrm{CO}_{2}$ atmosphere, $37^{\circ} \mathrm{C}$. Cells were split when confluence was reached, approximately every 7-9 days. Release from PC12 cells was measured with the same method as used for the cell model with two exceptions (1) the electrode was placed on the top of the PC12 cell rather than on the side and (2) exocytosis was stimulated by flushing the cell for 5 seconds with a depolarizing buffer containing $100 \mathrm{mM} \mathrm{K}^{+}$ (Picospritzer II; General Valve Instruments, Fairfield, NJ).

1. Chan, Y. H. M., van Lengerich, B. \& Boxer, S. G. Lipid-anchored DNA mediates vesicle fusion as observed by lipid and content mixing. Biointerphases $\mathbf{3}$, FA17-FA21 (2008).

2. Chen, Y. A. \& Scheller, R. H. Snare-mediated membrane fusion. Nat. Rev. Mol. Cell Biol. 2, 98-106 (2001).

3. Stengel, G., Zahn, R. \& Hook, F. DNA-induced programmable fusion of phospholipid vesicles. J. Am. Chem. Soc. 129, 9584 (2007).

4. Stengel, G., Simonsson, L., Campbell, R. A. \& Hook, F. Determinants for membrane fusion induced by cholesterol-modified DNA zippers. J. Phys. Chem. B $112,8264-8274$ (2008)

5. Simonsson, L., Jonsson, P., Stengel, G. \& Hook, F. Site-Specific DNA-Controlled Fusion of Single Lipid Vesicles to Supported Lipid Bilayers. ChemPhysChem 11, 1011-1017 (2010)

6. Rawle, R. J., van Lengerich, B., Chung, M., Bendix, P. M. \& Boxer, S. G. Vesicle Fusion Observed by Content Transfer across a Tethered Lipid Bilayer. Biophys. J. 101, L37-L39 (2011)

7. Wightman, R. M. et al. Temporally resolved catecholamine spikes correscpond to single vesicle release from individual chromaffin cells. Proc. Natl. Acad. Sci. U.S.A. 88, 10754-10758 (1991).

8. Mosharov, E. V. \& Sulzer, D. Analysis of exocytotic events recorded by amperometry. Nat. Methods 2, 651-658 (2005)

9. Karlsson, M. et al. Electroinjection of colloid particles and biopolymers into single unilamellar liposomes and cells for bioanalytical applications. Anal. Chem. 72, 5857-5862 (2000).

10. Cans, A. S. et al. Artificial cells: Unique insights into exocytosis using liposomes and lipid nanotubes. Proc. Natl. Acad. Sci. U.S.A. 100, 400-404 (2003).

11. Pfeiffer, I. \& Hook, F. Bivalent cholesterol-based coupling of oligonucletides to lipid membrane assemblies. J. Am. Chem. Soc. 126, 10224-10225 (2004).

12. Cans, A. S. et al. Amperometric detection of exocytosis in an artificial synapse. Anal. Chem. 75, 4168-4175 (2003).

13. Amatore, C., Oleinick, A. I. \& Svir, I. Reconstruction of Aperture Functions during Full Fusion in Vesicular Exocytosis of Neurotransmitters. ChemPhysChem 11, 159-174 (2010).

14. Montesinos, M. S. et al. The crucial role of chromogranins in storage and exocytosis revealed using chromaffin cells from chromagranin A null mouse. J. Neurosci. 28, 3350-3358 (2008)

15. Tabares, L., Lindau, M. \& de Toeldo, G. A. Relationship between fusion pore opening and release during mast cell exocytosis studied with patch amperometry. Biochem. Soc. Trans. 31, 837-841 (2003).
16. Schweigert, N., Zehnder, A. J. B. \& Eggen, R. I. L. Chemical properties of catechols and their molecular modes of toxic action in cells, from microorganisms to mammals. Environ Microbiol. 3, 81-91 (2001).

17. Orlowski, A. et al. Strong preferences of daopamine and L-dopa towards lipid head group: importance of composition and implication for neurotransmitter metabolism. J. Neurochem. 122, 681-690 (2012).

18. Olsen, F., Hunt, C. A., Szoka, F. A., Vail, W. J. \& Papahadjopoulos, D. Preparation Of liposomes of defined size distribution through polycarbonate membranes. Biochim. Biophys. Acta 557, 9-23 (1979).

19. Rodahl, M., Hook, F., Krozer, A., Brzezinski, P. \& Kasemo, B. Quartz-crystal microbalance setup for frequency and q-factor measurements in gaseous and liquid environments. Rev. Sci. Instrum. 66, 3924-3930 (1995).

20. Papahadjopoulos, D., Nir, S. \& Düzgünes, N. Molecular Mechanisms of CalciumInduced Membrane Fusion. J. Bioenerg. Biomembr. 22, 157-179 (1990).

21. Altenbach, C. \& Seelig, J. Ca ${ }^{2+}$ Binding to phosphatidylcholine bilayers as studied by deuterium magnetic resonance. Evidence for the formation of a $\mathrm{Ca}^{2+}$ complex with two phospholipid molecules. Biochemistry 23, 3913-3920.

22. Amatore, C. et al. Relationship between amperometric pre-spike feet and secretion granule composition in Chromaffin cells: An overview. Biophys. Chem 129, 181-189 (2007).

23. Detoledo, G. A., Fernandezchacon, R. \& Fernandez, J. M. Release of secretory products during transient vesicle fusion. Nature 363, 554-558 (1993).

24. Staal, R. G. W., Mosharov, E. V. \& Sulzer, D. Dopamine neurons release transmitter via a flickering fusion pore. Nat. Neurosci. 7, 341-346 (2004)

25. Amatore, C. et al. Regulation of exocytosis in chromaffin cells by trans-insertion of lysophosphatidylcholine and arachidonic acid into the outer leaflet of the cell membrane. ChemBioChem. 7, 1980-2003 (2006).

26. Uchiyama, Y., Maxson, M. M., Sawada, T., Nakano, A. \& Ewing, A. G. Phospholipid mediated plasticity in exocytosis observed in PC12 cells. Brain Res. 1151, 46-54 (2007).

27. Mayer, L. D., Hope, M. J., Cullis, P. R. \& Janoff, A. S. Solute distributions and trapping efficiencies observed in freeze-thawed multilamellar vesicles. Biochim. Biophys. Acta 817, 193-196 (1985).

28. Karlsson, M. et al. Micropipet-assisted formation of microscopic networks of unilamellar lipid bilayer nanotubes and containers. Langmuir 17, 6754-6758 (2001).

29. Kozminski, K. D., Gutman, D. A., Davila, V., Sulzer, D. \& Ewing, A. G. Voltammetric and pharmacological characterization of dopamine release from single exocytotic events at rat pheochromocytoma (PC12) cells. Anal. Chem. 70, 3123-3130 (1998).

\section{Acknowledgements}

This work was supported by the Swedish Research Council Grant-IDs: 80348401, 80348402, 349-2007-8680 and 2010-5063. The authors would like to thank Andrew Ewing for supplying PC12 cells and for helpful scientific discussion as well as Lisa Mellander for her expertise in handling the cells.

\section{Author contributions}

L.S. and M.E.K. performed all experiments and analysed and interpreted data; L.S. prepared the LUVs. M.E.K. performed the PC12 measurement and fabricated the microelectrodes; R.T. performed the simulations; A.S.C., F.H. and L.S. designed the experiments for the development of the artificial cell model; L.S. and M.E.K. wrote the main part of the manuscript together with A.S.C.; all authors read and approved the manuscript.

\section{Additional information}

Supplementary Information accompanies this paper at http://www.nature.com/ scientificreports

Competing financial interests: The authors declare no competing financial interests. License: This work is licensed under a Creative Commons Attribution-NonCommercial-NoDerivs 3.0 Unported License. To view a copy of this license, visit http://creativecommons.org/licenses/by-nc-nd/3.0/

How to cite this article: Simonsson, L., Kurczy, M.E., Trouillon, R., Hook, F. \& Cans, A A functioning artificial secretory cell. Sci. Rep. 2, 824; DOI:10.1038/srep00824 (2012). 